\title{
Terminal Differentiation of RBL-2H3 cells in vitro
}

\author{
Luisa Otalora $^{1}$, Jonathan Blaize ${ }^{1}$, William J. L'Amoreaux ${ }^{1,2}$ and Robert Corin ${ }^{1}$ \\ ${ }^{1}$ The College of Staten Island, The City University of New York, 2800 Victory Blvd. \\ Staten Island, New York 10314 \\ ${ }^{2}$ Advanced Imaging Facility of the College of Staten Island, 2800 Victory Blvd., Staten \\ Island, NY 10314
}

RBL-2H3 cells are neoplastically transformed cells that express some phenotypic characteristic of mature mast cells (MC) including the FceR1 type IgE receptor. Mature rat MC's are composed of mucosal mast cell (MMC) and connective tissue mast cell (CTMC) subpopulations. MMC and CTMC are functionally distinguished by responsiveness to extracellular ligands that elicit secretion. RBL cells can transdifferentiate to the CTMC phenotype by treatment with quercetin $(\mathrm{Q})$, a broad spectrum kinase inhibitor. Preliminary experiments in our lab have demonstrated that high concentrations of $\mathrm{Q}(30-60 \mu \mathrm{M})$ does not cause terminal differentiation as determined by a high cloning efficiency (nearly control efficiency) of RBL-2H3. In this study we examined the effects of butyrate, an inhibitor of histone deacetylase, upon growth, morphology, and terminal differentiation of RBL-2H3 cells in vitro. Butyrate was chosen since it is known to induce differentiation of transformed cells in vitro. Our goal is to understand the loss of proliferative capacity as a component of commitment to terminal differentiation.

After 24 and 48 hours of treatment with $2.0 \mathrm{mM}$ butyrate, adherent RBL cells exhibited full metabolic activity and $100 \%$ viability (as demonstrated by dye exclusion) compared to parallel control cultures. However, the butyrate cells treated for $24 \mathrm{hr}$ displayed loss of proliferative potential as evidenced by $\sim 80 \%$ reduction of cloning efficiency (compared to control cells) after butyrate removal (not shown).

Microscopic examination of living cells after treatment with $2.0 \mathrm{mM}$ butyrate for 24 hours revealed striking morphological changes (Fig. 1). The cells developed numerous processes and many cells accumulated large vesicular structures not evident in control cells. Although these distinct morphologies of the butyrate treated cells were not observed in matched control cultures, the induced morphologies are not unique to butyrate treated cultures. For the experiment shown cultures were inoculated at low density and the control cells were still growing exponentially at the termination of the experiment. Similar morphologies were observed, albeit in a much smaller portion of the population, in untreated cells that have grown to saturation density and ceased exponential growth. However, we do not believe the growth arrest caused by butyrate is solely responsible since Q mediated growth arrest of low density cultures does not result in appearance of the butyrate morphology (not shown). 
Upon more prolonged incubation with butyrate $(48 \mathrm{hr})$ a progression to a more flattened morphology relative to control cells was noted and visualized with scanning electron microscopy (Fig. 2). As previously noted, similar morphologies were observed, albeit in a much smaller portion of the population, in untreated cells that have grown to saturation density and ceased exponential growth. However, we do not believe growth arrest is solely responsible fore the majority of cells assuming this flattened morphology. Finally, we also observed that treatment with butyrate increases the amount of autofluorescent secretory granules (as observed by confocal microscopy; Figure 3 ).

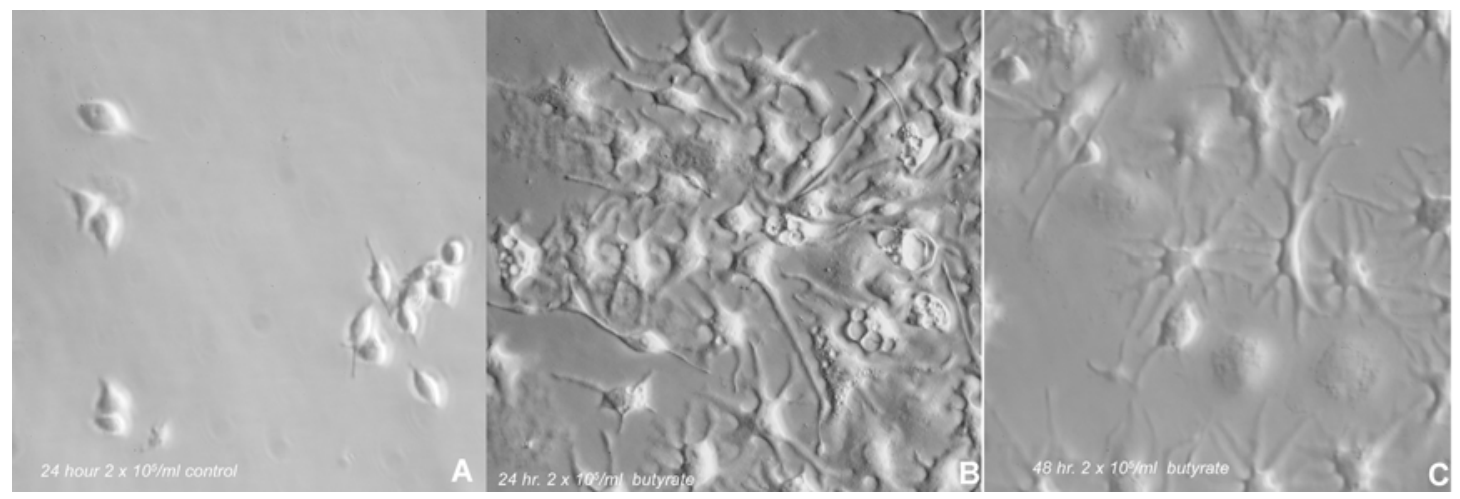

Figure 1. Control cells (A) exhibit a markedly different morphology compared to cells treated with $2 \mathrm{mM}$ butyrate for $24 \mathrm{hr}$ (B) or $48 \mathrm{hr}$ (C).
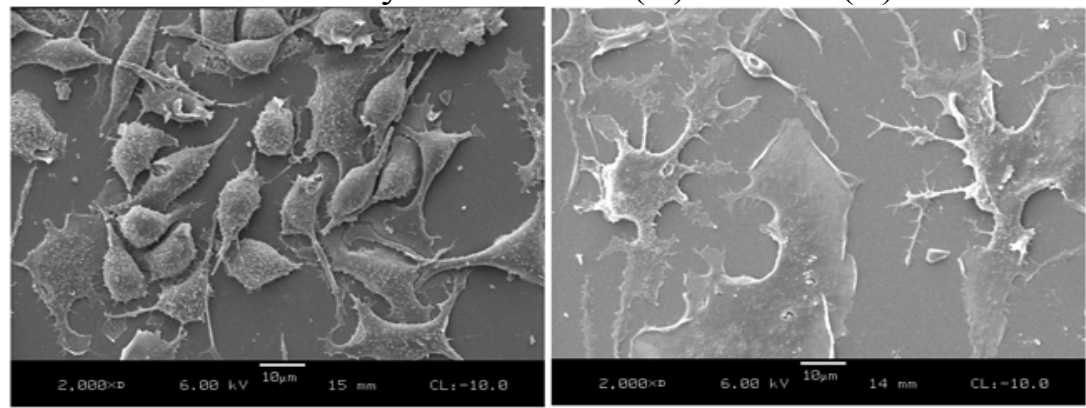

Figure 2. Scanning electron micrographs of control (left) and butyrate-treated RBL-2H3. Control cells remain slightly rounded whereas butyrate treatment induces differentiation to a morphology reminiscent of dendritic cells.

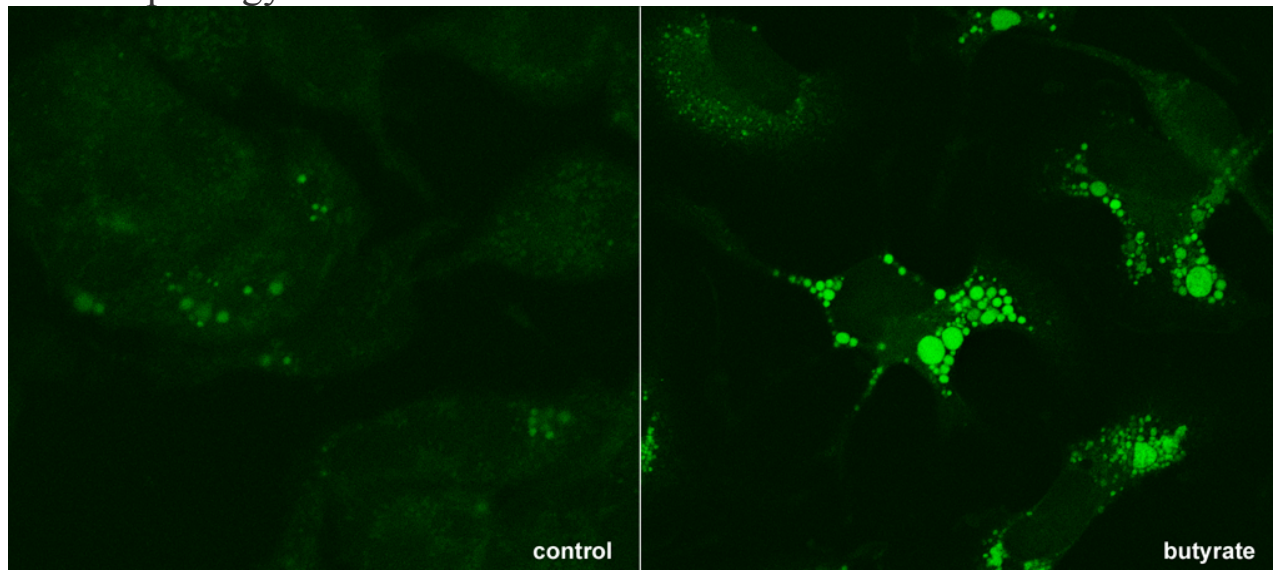

Figure 3. CLSM images of control vs. butyrate-treated cells. There is a marked increase in the number of secretory granules in the butyrate-treated cells. 